\title{
Geometrical influences on neoclassical magnetohydrodynamic tearing modes
}

\author{
S. E. Kruger, C. C. Hegna, and J. D. Callen \\ Department of Nuclear Engineering and Engineering Physics, 1500 Engineering Drive Madison, \\ Wisconsin 53706-1687
}

(Received 5 August 1997; accepted 4 November 1997)

\begin{abstract}
The influence of geometry on the pressure drives of nonideal magnetohydrodynamic tearing modes is presented. In order to study the effects of elongation, triangularity, and aspect ratio, three different machines are considered to provide a range of tokamak configurations: Tokamak Fusion Test Reactor (circular) [Fusion Technol. 21, 1324 (1992)], DIII-D (D-shaped) [Plasma Physics and Controlled Nuclear Fusion Research 1986 (International Atomic Energy Agency, Vienna, 1987), Vol. 1, p. 159], and Pegasus (extremely low aspect ratio) [Fonck et al., Bull. Am. Phys. Soc. 41, 1400 (1996)]. For large aspect ratio tokamaks, shaping does very little to influence the pressure gradient drives, while at low aspect ratios, a very strong sensitivity to the profiles is found. In particular, this sensitivity is connected to the strong dependence on the magnetic shear. This suggests that at low aspect ratio it may be possible to stabilize neoclassical tearing modes by a flattening the $q$ profile near low order rational surfaces (e.g., $q=2 / 1$ ) using a combination of shaping and localized current drive, whereas at large aspect ratio it is more difficult. (C) 1998 American Institute of Physics. [S1070-664X(98)01602-4]
\end{abstract}

\section{INTRODUCTION}

The success of magnetic fusion experiments in obtaining high plasma pressure discharges has largely been due to the design and operation of the experiments around the stability of the discharge to ideal magnetohydrodynamic (MHD) instabilities. ${ }^{1}$ As experiments continue to operate near the ideal stability limit, MHD instabilities that develop on a time scale slower than the ideal MHD time scale and are associated with magnetic reconnection determine the constraints and limitations of the discharges. Of particular concern in recent years have been tearing-type modes, which either cause the plasma to disrupt or degrade its confinement.,

Many of the plasmas with the observed tearing-type modes have profiles that are believed to be stable when using resistive MHD theory (i.e., $\Delta^{\prime}<0^{4}$ ). In order to explain the appearance of these instabilities, the effect of the perturbed bootstrap current in the generalized Ohm's law has been studied. ${ }^{5-7}$ The bootstrap current is a parallel current generated from the viscous damping of the poloidal component of the electron diamagnetic current. Because this current is proportional to the gradient of the pressure, a pressure perturbation leads to a perturbed parallel current. These current perturbations, caused by a "seed island" that flattens the pressure profile, are destabilizing for the bootstrap current when $d p / d q<0$, where $p$ is the equilibrium pressure and $q$ is the inverse rotational transform. Neoclassical tearing modes have been shown to agree with theory ${ }^{3,8}$ and have been seen in the Tokamak Fusion Test Reactor (TFTR), ${ }^{3,8}$ DIII-D, ${ }^{9}$ Axisymmetric Divertor Experiment-Upgrade (ASDEX-U), ${ }^{10}$ COMPASS-D, ${ }^{11}$ and Japan Torus-60 Upgrade (JT-60U). ${ }^{12}$ They are predicted to be problematic for the International Thermonuclear Experimental Reactor
(ITER), ${ }^{12}$ since they may lead to a beta limit below that required for ignition.

The Pfirsch-Schlüter current, or return current, is a parallel current required to maintain quasineutrality in a torus. Because the divergence of the diamagnetic current is nonzero in a torus, solving for the parallel current from $\boldsymbol{\nabla} \cdot \mathbf{J}=0$ yields a parallel current proportional to the pressure gradient. Since pressure perturbations arise that are consistent with magnetic island formation, resistive interchange effects enter into the parallel current profile. ${ }^{13}$ When $d p / d q<0$, the resistive interchange (or Glasser ${ }^{14}$ ) effect is stabilizing in the linear regime and the nonlinear regime. ${ }^{13}$ At large aspect ratio, the order $\beta$ resistive interchange effect is small relative to the order $\sqrt{\epsilon} \beta_{P}$ neoclassical effect and hence the stabilizing effect is small in present tokamaks. At smaller aspect ratios, the strong toroidicity implies a larger Pfirsch-Schlüter current and the stabilizing effect increases. At very low aspect ratio, the resistive interchange and neoclassical effects become comparable, creating the possibility of profiles that accentuate the stabilizing resistive interchange effect, and therefore have very favorable nonideal MHD stability properties at high $\beta$.

In this paper, the influence of geometry upon the drives for nonideal tearing modes is explored. In Sec. II, the theory of neoclassical tearing modes is reviewed and the forms of the drives are presented. In Sec. III, the drive behavior is examined under a variety of shaping conditions for a TFTR equilibrium, a DIII-D equilibrium, and a Pegasus Extremely Low Aspect Ratio Tokamak (ELART) equilibrium. In Sec. IV we present a method to determine the marginal stability of the neoclassical tearing mode and typical stability curves for the three devices are shown. In Sec. V we summarize the results. 


\section{THEORY}

An analytic model for magnetic island dynamics can be derived using a modified version of the nonlinear Rutherford theory. ${ }^{15}$ In this model, the evolution of the magnetic island width $w$ is governed by ${ }^{7,13}$

$$
\frac{d}{d t} w=1.22 \frac{\eta_{\mathrm{nc}}}{\mu_{0}}\left(\Delta^{\prime}+4.6 \frac{D_{\mathrm{nc}}+D_{R}}{w}\right),
$$

where $\eta_{\mathrm{nc}}$ is the neoclassical resistivity, $\Delta^{\prime}$ is the resistive MHD matching parameter, ${ }^{4} D_{\mathrm{nc}}$ is the term representing the neoclassical drive, and $D_{R}$ is the term representing the Pfirsch-Schlüter current drive. ${ }^{14}$ This equation is derived using a neoclassical version of the parallel Ohm's law and an assumption that the pressure in the vicinity of an island has equilibrated along magnetic field lines. ${ }^{5}$ In the limit of zero pressure, the standard Rutherford equation ${ }^{15}$ is recovered. Other effects, which may play an important role at a small island width, may be included, ${ }^{16-19}$ but are not considered here since we are concerned with stability properties above the nonlinear "seed island" threshold. For the typical case when the discharge is stable to resistive tearing modes $\left(\Delta^{\prime}\right.$ $<0$ ), the magnetic island grows to a saturated island width, given by

$$
w_{\mathrm{sat}}=4.6\left(\frac{D_{\mathrm{nc}}+D_{R}}{-\Delta^{\prime}}\right) .
$$

Because the combination of $D_{\mathrm{nc}}$ and $D_{R}$ determine the saturated island width, we will investigate the geometrical influences on these terms. Also, since $\Delta^{\prime} \sim-2 m / r_{s} \propto m$, only the lowest $m$ modes have large enough island widths to be of concern, ${ }^{3}$ and we will primarily be concerned with $D_{\text {nc }}$ $+D_{R}$ at low-order rational surfaces $(q=2 / 1,3 / 1,3 / 2,5 / 2$, etc. $)$

The resistive interchange parameter, $D_{R}$ or Glasser term, is given by ${ }^{14}$

$$
\begin{aligned}
& D_{R}=D_{I}+(H-1 / 2)^{2}, \\
& D_{I}=E+F+H-1 / 4, \\
& E=C_{1}\left(p_{\psi} / q_{\psi}^{2}\right)+C_{2}\left(p_{\psi} / q_{\psi}\right), \\
& F=C_{3}\left(p_{\psi} / q_{\psi}\right)^{2}, \\
& H=C_{4}\left(p_{\psi} / q_{\psi}\right),
\end{aligned}
$$

where $p_{\psi}=d p / d \psi, q_{\psi}=d q / d \psi$, and $\psi$ is the poloidal flux. The $C_{i}$ coefficients depend upon the geometry and equilibrium and are given by

$$
\begin{aligned}
& C_{1}=\left(\frac{V_{\psi}}{2 \pi}\right)^{2}\left(\frac{I_{\psi}}{I}-\left\langle\frac{d}{d_{\psi}} \ln \left(R^{2}\right)\right\rangle\right), \\
& C_{2}=\frac{V_{\psi}}{2 \pi}\left(\frac{I}{\left\langle B^{2}\right\rangle}\left\langle\frac{B^{2}}{|\nabla \psi|^{2}}\right\rangle-\frac{V_{\psi}}{2 \pi q}\right), \\
& C_{3}=\left(\frac{V_{\psi}}{2 \pi}\right)^{2}\left(\left\langle\frac{R^{2}}{|\nabla \psi|^{2}}\right\rangle\left\langle\frac{B^{2}}{|\nabla \psi|^{2}}\right\rangle-I^{2}\left\langle\frac{1}{|\nabla \psi|^{2}}\right\rangle^{2}\right), \\
& C_{4}=\frac{V_{\psi}}{2 \pi} I\left(\left\langle\frac{1}{|\nabla \psi|^{2}}\right\rangle-\frac{1}{\left\langle B^{2}\right\rangle}\left\langle\frac{B^{2}}{|\nabla \psi|^{2}}\right\rangle\right),
\end{aligned}
$$
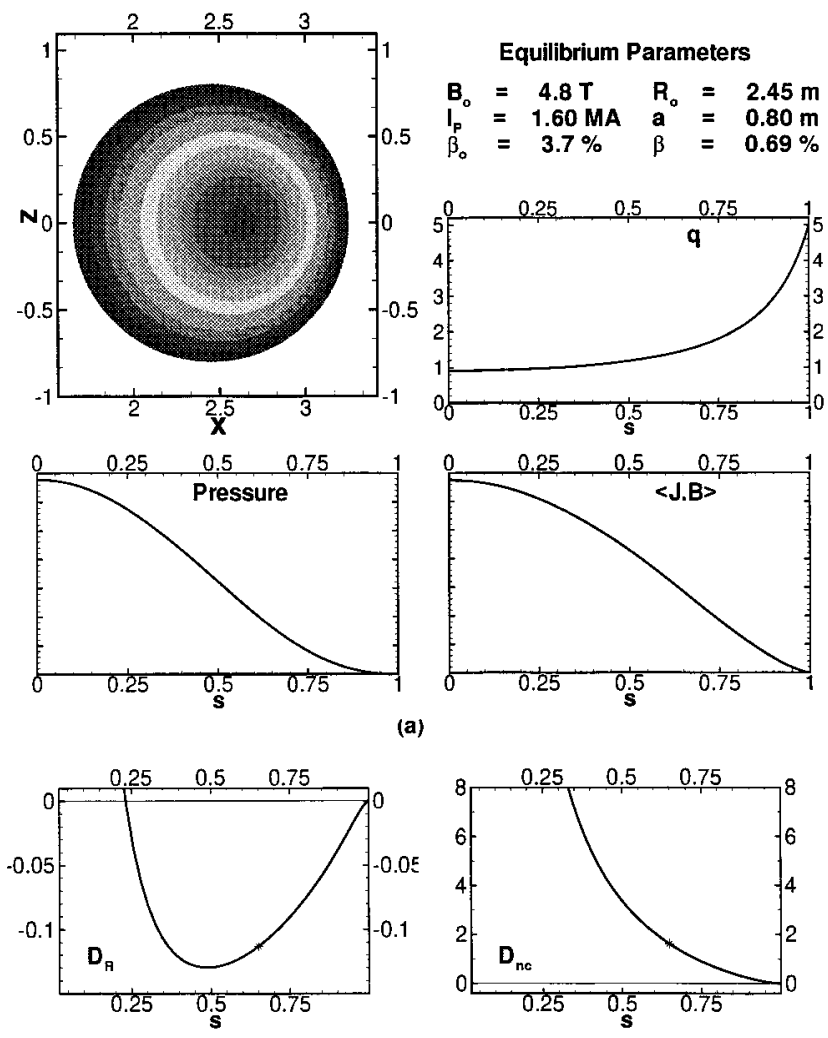

(b)

FIG. 1. Reference TFTR equilibrium based on shot 66886 showing relevant profiles and parameters in (a). As seen in (b), the resistive interchange term is negligible compared to the neoclassical term at the $3 / 2$ rational surface, which is marked by an asterisk. Plots of flux surface quantities are plotted using $s \equiv \sqrt{\left(\psi-\psi_{\min }\right) /\left(\psi_{\max }-\psi_{\min }\right)}$ as the flux coordinate.

where $R$ is the major radius, $I$ is the major radius times the toroidal field, $V$ is the volume enclosed by a magnetic flux surface, and $\langle\cdots\rangle$ denotes an average over a flux surface. Because $D_{R}$ is typically negative for tokamak discharges, this effect is stabilizing for tearing modes, as can be seen in Eq. (1).

The neoclassical bootstrap current effects are quantified by the following term, which is in a form approximately valid $^{20-22}$ for arbitrary aspect ratio in the collisionless regime when temperature gradients and heat flows are neglected, and for $Z_{i}=1$ :

$$
D_{\mathrm{nc}}=-\frac{1.5 f_{t} / f_{c}}{1+1.5 f_{t} / f_{c}} \frac{q d p / d q}{\left\langle B_{\theta}^{2}\right\rangle / 2 \mu_{0}},
$$

where $f_{t}$ is the trapped particle fraction, $f_{c}$ is the circulating particle fraction, and $B_{\theta}$ is the poloidal magnetic field. At large aspect ratio $[1 / \sqrt{\epsilon}$, the trapped particle fraction can be approximated by $f_{t} \approx 1.46 \sqrt{\epsilon}$ and the numerical coefficient becomes $\left.^{22} 2.3 \sqrt{\epsilon} /(1+2.3 \sqrt{\epsilon})\right]$. In contrast to the Glasser term, the neoclassical term is destabilizing for conventional tokamak profiles, where $d p / d q<0$. We note that our analysis only uses the pressure gradient for the bootstrap drive rather than using the density and temperature gradients for each Maxwellian species and fast ions. Because the error in using this $1 \times 1$ transport matrix approximation frequently lies within experimental error, the approximation is valid to 

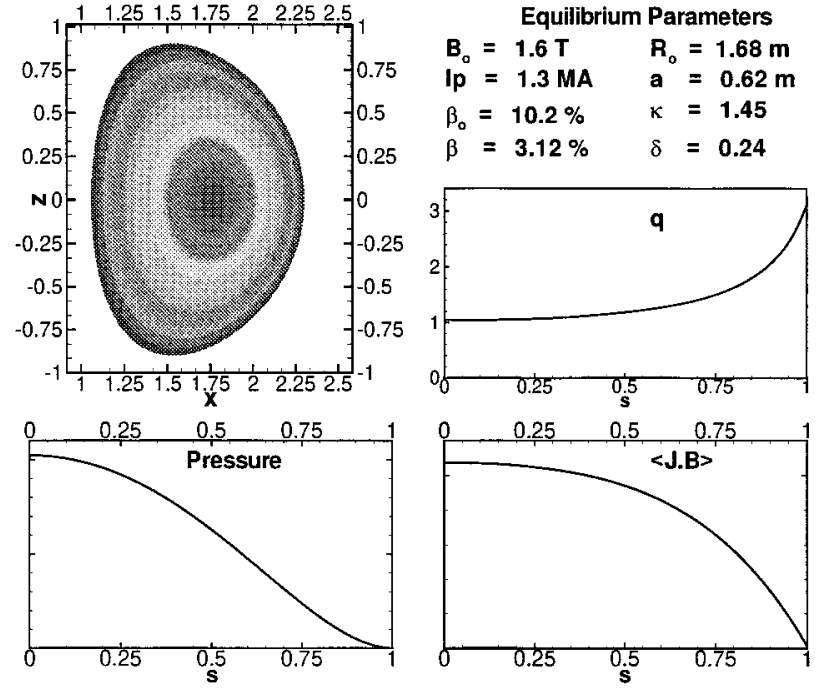

(a)
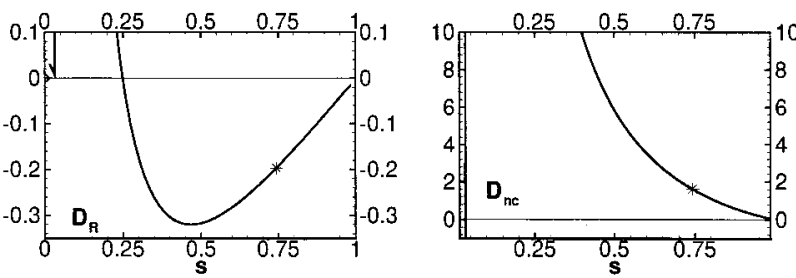

(b)

FIG. 2. (a) The DIII-D equilibrium used is an up-down symmetric version of a long-pulse, ITER-like discharge. (b) The resistive interchange term is approximately $10 \%$ at the $3 / 2$ rational surface, which is indicated by an asterisk.

give the dominant behavior. Differences in heating that result in fast ion populations with very different velocity space distributions will have an even smaller effect due to the small viscosity of fast ions.

We investigate the competition between the resistive interchange term and the neoclassical term for a variety of equilibria. The equilibrium code used is TOQ, written by R. L. Miller. The method used to calculate the circulating particle fraction is based on a method put forth by Lin-Liu. ${ }^{23}$

\section{GEOMETRY DEPENDENCE}

\section{A. TFTR equilibria}

A useful reference case to begin studying nonideal effects on tearing modes is TFTR because it is a conventional
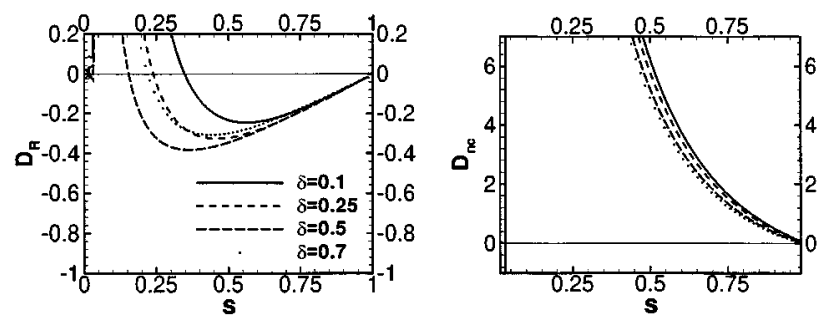

FIG. 3. For DIII-D equilibria, triangularity $(\delta)$ has very little influence on the pressure-gradient tearing mode drives.

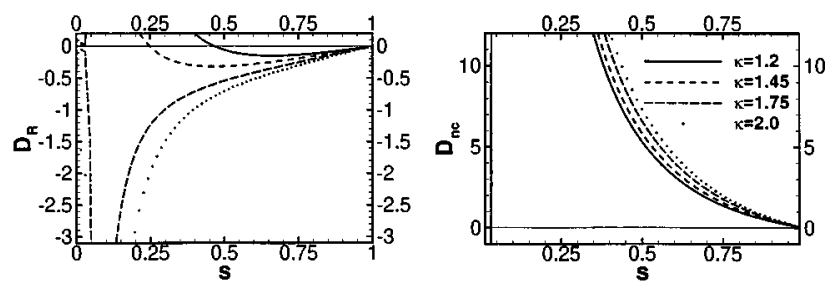

FIG. 4. Elongation $(\kappa)$ has relatively little effect on the pressure drives for DIII-D equilibria. Because both terms have a similar scaling, but mostly are of opposite sign, a plot of the sum of the two terms would show even less variation.

circular cross section, large aspect ratio device, and it was the first machine to use neoclassical tearing mode theory to explain experimental observations. ${ }^{3,8}$ The reference equilibrium, based on shot 66886, which had an $m / n=3 / 2$ mode, is shown in Fig. 1(a). In the figure, the flux surface quantities are plotted versus $s \equiv \sqrt{\left(\psi-\psi_{\min }\right) /\left(\psi_{\max }-\psi_{\min }\right)}$. As illustrated in Fig. 1(b), the resistive interchange term is approximately $5 \%$ of the neoclassical term. Typical of the conventional circular cross section, large aspect ratio tokamaks, the resistive interchange term is almost always negligible and the total pressure-gradient drive is destabilizing.

\section{B. DIII-D equilibria}

Equilibria in DIII-D differ from TFTR-type equilibria because shaping influences the two drive mechanisms and they have a slightly smaller aspect ratio. To study the influence of elongation and triangularity for these types of equilibria, a reference case was chosen and scans were performed holding the total current, the vacuum toroidal field, the pressure profile, and the profile of the flux surface average of the dot product of the current density and magnetic field, $\langle\overrightarrow{\mathbf{J}} \cdot \overrightarrow{\mathbf{B}}\rangle$, fixed. The reference equilibrium is shown in Fig. 2(a), which is an up-down symmetric equilibrium reconstruction of a long-pulse, ITER-like discharge that exhibited an $m / n=3 / 2$ mode. As seen in Fig. 2(b), the shaped tokamak has a Glasser term that is approximately $10-20 \%$ of the neoclassical term.

As shown in Figs. 3 and 4, shaping does relatively little to influence the resistive interchange and bootstrap terms in DIII-D. Because the scaling of the resistive interchange and bootstrap terms are similar but of opposite sign, plots of the sum of $D_{R}$ and $D_{\text {nc }}$ show even less variation with the shaping parameters. These results suggest that shaping will play a very little role in the efforts to stabilize these modes in DIII-D. However, if the magnetic shear becomes very small somewhere in the plasma the resistive interchange term can dominate there-an effect that is much clearer for very low aspect ratios, as explained in the next section.

\section{Pegasus equilibria}

The Pegasus ELART, under construction at the University of Wisconsin-Madison, provides an interesting case to explore some extremes of behavior due to its extremely low aspect ratio $(A \lesssim 1.2)$. The reference equilibrium is shown in Fig. 5(a). The profiles shown are typical of the profiles pro- 

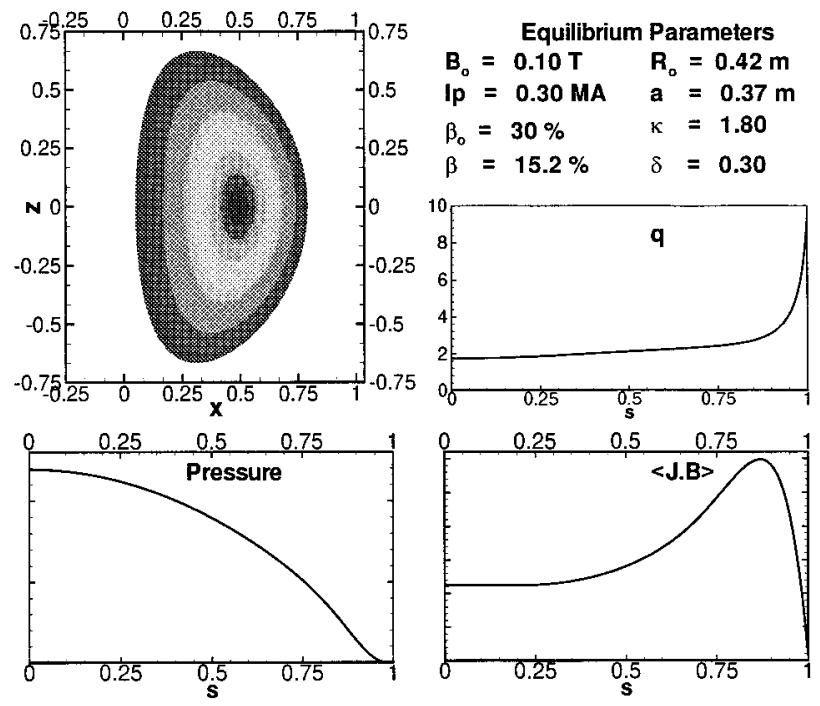

(a)
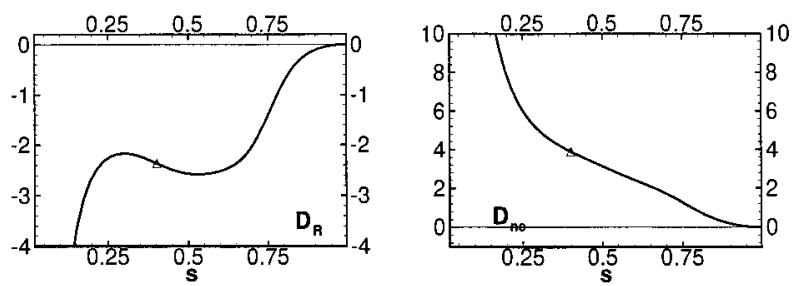

(b)

FIG. 5. A Pegasus equilibrium shown in (a) uses typical profiles for low aspect ratio tokamaks. The two pressure drives are of comparable magnitude at the $2 / 1$ surface, which is indicated by the triangles in (b).

posed to achieve high $\beta$ in low aspect ratio devices. ${ }^{24,25}$ The reference case shown here is stable to ideal MHD modes up to $\beta=48 \%$; however, a more moderate $\beta$ was chosen for the reference case. Scans were performed on triangularity and elongation using the same procedure as for the DIII-D equilibria.

The two drive terms scale with $\beta$ and the magnetic shear $\hat{s}$ as

$$
D_{\mathrm{nc}} \sim \frac{\sqrt{\epsilon} \beta_{\theta}}{\hat{s}} ; \quad D_{R} \sim \frac{\beta}{\hat{s}^{2}},
$$

in which $\hat{s} \equiv 2 V / q(d q / d V)$. In low aspect ratio tokamaks, where $\beta$ is large and the shear is small, it possible that the two terms will be of comparable size and larger than the large aspect ratio case, as seen in Fig. 5(b). In contrast to the high aspect ratio case, a dramatic effect on stability due to shaping can be seen in Figs. 6 and 7, where we have also plotted the $q$ profiles and the sum of $D_{R}$ and $D_{\mathrm{nc}}$.

As triangularity increases, shear increases in the center but slightly decreases at the edge, as seen in Fig. 6. Stabilization at the lowest triangularity is possible where the shear decreases to the point that the resistive interchange term dominates. However, the flat $q$ profile also leads to a sensitivity of the location of the low-order rational surfaces. Because the sum of the bootstrap and resistive interchange drive terms increases as one moves toward the center of the plasma, the value at a particular rational surface may actually increase. In Fig. 6, the location of the $m / n=2 / 1$ rational
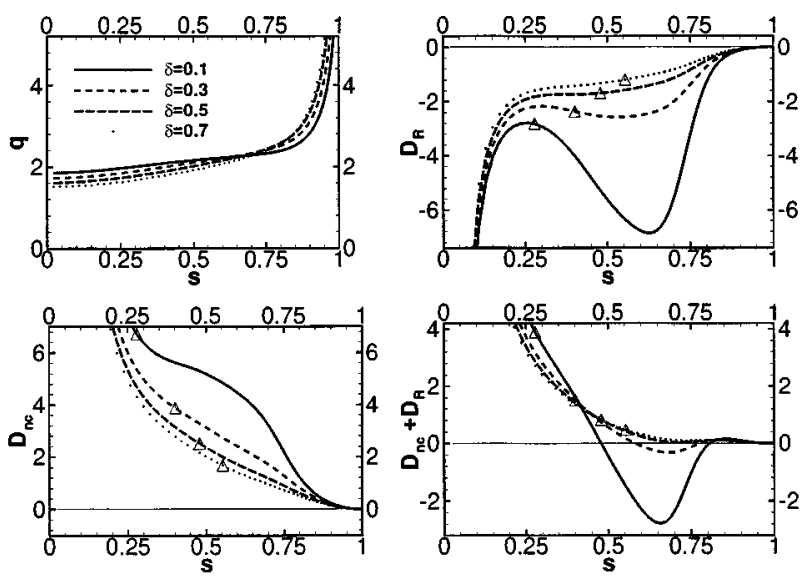

FIG. 6. Varying the triangularity $(\delta)$ for the low aspect ratio Pegasus equilibrium produces a more dramatic effect at normal, high aspect ratio (cf. Fig. 1). When the shear is low enough, the total drive may be stabilizing. A particular rational surface, such as the $2 / 1$ indicated, may, however, become more unstable.

surface is shown. Even though the low triangularity case shows a large region of stabilization, the pressure-gradient drive at this rational surface is at its maximum for this case.

As elongation increases, the shear is increased, leading to a reduction in the neoclassical drive, as seen in Fig. 7. The total drive, however, is relatively unaffected, except at the lowest elongation, where the shear is very small. Because the current was held fixed as the area increased with elongation, the entire $q$ profile increases and the location of a particular rational surface can vary over a rather large distance because of the flat $q$ profile in the center. Like the triangularity scan, this scan illustrates how the behavior of neoclassical tearing modes in LART's is very sensitive to the profiles used.

\section{STABILITY DIAGRAMS FOR THE NEOCLASSICAL TEARING MODES}

From the scans in triangularity and elongation at low aspect ratio, it is clear that one can stabilize the neoclassical
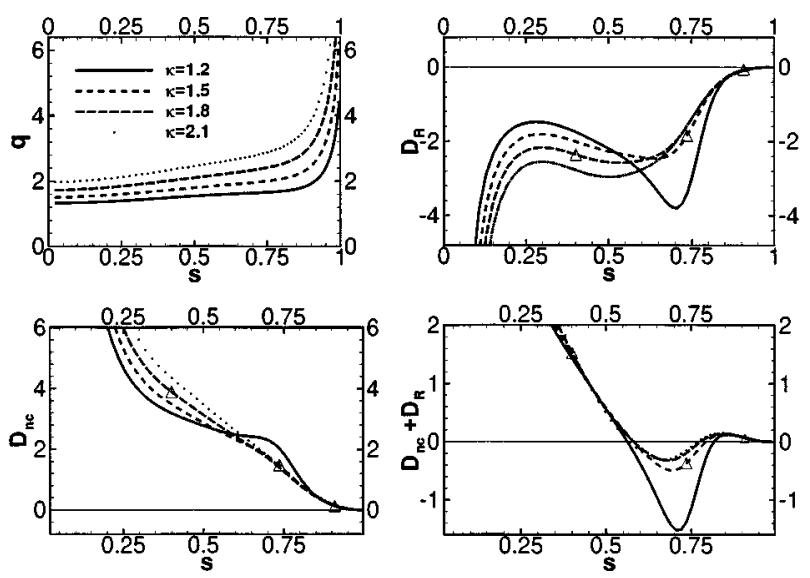

FIG. 7. Elongation has very little effect on the Pegasus equilibrium, except at low elongation, where the shear is extremely low. The location of a low-order rational surface, such as the $2 / 1$, which is marked with a triangle, varies widely because of the flat $q$ profile in the core. 

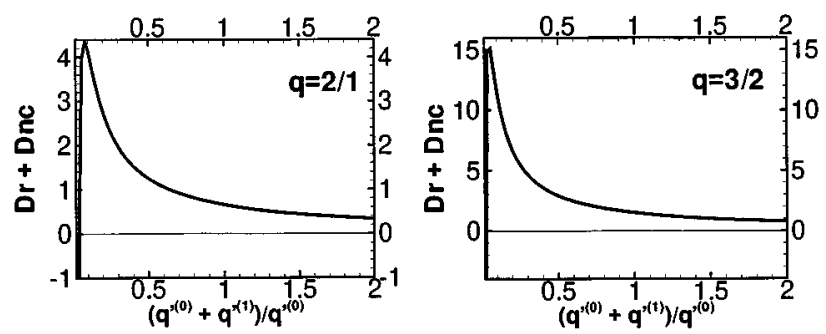

FIG. 8. For TFTR equilibria, the stability curves illustrate that shear reversal, represented by the negative $x$ axis, is necessary to eliminate the drives for these modes for both the $2 / 1$ and $3 / 2$ rational surfaces.

tearing mode if the shear at a low-order rational surface is small enough. One would like to quantify how flat the $q$ profile must be to stabilize the modes, because using localized current drive to flatten the $q$ profile would be an attractive way of stabilizing these modes.

To quantify this behavior, and to gain a deeper understanding of these modes, an analysis similar to that used by Greene and Chance ${ }^{26}$ was performed. In this type of analysis, a class of equilibria is generated by introducing small localized perturbations to the equilibrium quantities, but zerothorder corrections to the gradients:

$$
\begin{aligned}
& p(\psi)=p(\psi)^{(0)}+\mu p(y)^{(1)} ; \\
& \frac{d p}{d \psi}=\frac{d p}{d \psi}(\psi)^{(0)}+\frac{d p}{d y}(y)^{(1)}, \\
& q(\psi)=q(\psi)^{(0)}+\mu q(y)^{(1)} ; \\
& \frac{d q}{d \psi}=\frac{d q}{d \psi}(\psi)^{(0)}+\frac{d q}{d y}(y)^{(1)},
\end{aligned}
$$

where $y=\psi / \mu, \mu \ll 1$, and the superscripts denote the order of the perturbation. By examining the case $p(y)^{(1)}=0$, the sensitivity of the bootstrap and the resistive interchange terms to variations in the magnetic shear can be expressed in terms of the new equilibrium as

$$
\begin{aligned}
D_{\mathrm{nc}}+D_{R}= & \frac{q^{\prime(0)^{2}}}{\left(q^{\prime(0)}+q^{\prime(1)}\right)^{2}}\left(D_{I}^{(0)}+\frac{1}{4}+H^{(0)^{2}}\right) \\
& +\frac{q^{\prime(0)}}{\left(q^{(0)}+q^{\prime(1)}\right)}\left(D_{\mathrm{nc}}^{(0)}-H^{(0)}\right),
\end{aligned}
$$

where $D_{\mathrm{nc}}^{(0)}, D_{I}^{(0)}$, and $H^{(0)}$ are the stability parameters for the original equilibria and primes denote differentiation with respect to the dependent variable. The stability of the perturbed equilibria may now be evaluated using the stability parameters of the unperturbed equilibra. Plotting $D_{\mathrm{nc}}+D_{R}$ vs $\left(q^{\prime(0)}+q^{\prime(1)}\right) / q^{\prime(0)}$ generates the stability curve for pressure-gradient-driven MHD tearing modes.

In Fig. 8, the stability curves for two rational surfaces for the TFTR equilibria are shown. The point where $\left(q^{\prime(0)}\right.$ $\left.+q^{\prime(1)}\right) / q^{\prime(0)}=1$ corresponds to the equilibrium shown in Fig. 1. To the left of this point is weaker shear and to the right is increased shear. As can be seen in the figure, at weaker shear the drive for the neoclassical tearing mode actually increases until it is eliminated near the point of reverse
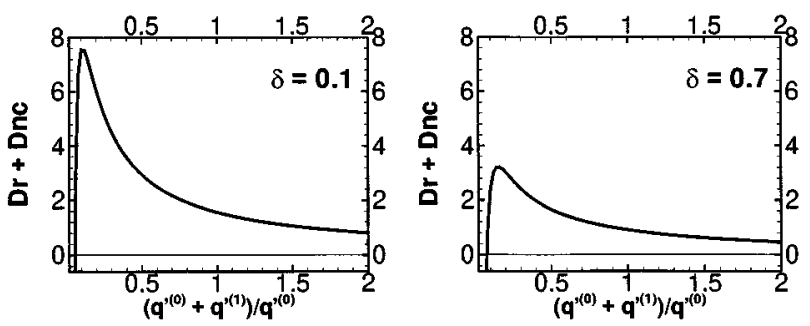

FIG. 9. For DIII-D equilibria, triangularity lowers the equilibrium value only slightly at the $q=3 / 2$ rational surface. Although the peak in the stability curve is lower at higher triangularity, the drives are still only stabilizing near shear reversal.

shear, which corresponds to the negative $x$ axis. For this case, the practical stability condition corresponds to having reversed magnetic shear. Also note that the peak of the curve is higher at lower $q$ values. This is true for all of the rational surfaces in the plasma and is true for the DIII-D cases as well.

In Fig. 9, the stability curves for the $q=3 / 2$ rational surface at different values of triangularity in DIII-D are shown. As in the TFTR case, stabilization by locally flattening the $q$ profile appears difficult, although it would be easier at higher triangularity. For the elongation scans, a similar behavior is seen, although it is at lower elongation that one sees the decrease in the peak of $D_{R}+D_{\mathrm{nc}}$. For both TFTR and DIII-D, the drives decrease by increasing the shear.

The stability diagrams are especially useful in studying neoclassical tearing modes in LART's. Figure 10 shows the stability diagram for two rational surfaces for the equilibrium shown in Fig. 5. Because only a little flattening of the $q$ profile will result in eliminating the drive for the $5 / 2$ mode, it is marginally stable. For the $2 / 1$ mode, increasing the shear decreases the drive very little; however, a region of stability develops for sufficiently small magnetic shear. In the inner core region where the shear is rather low, the shape of the curves are very sensitive to the specific profiles used; however, in the edge, where the shear is higher, the curves are similar to the large aspect ratio case.
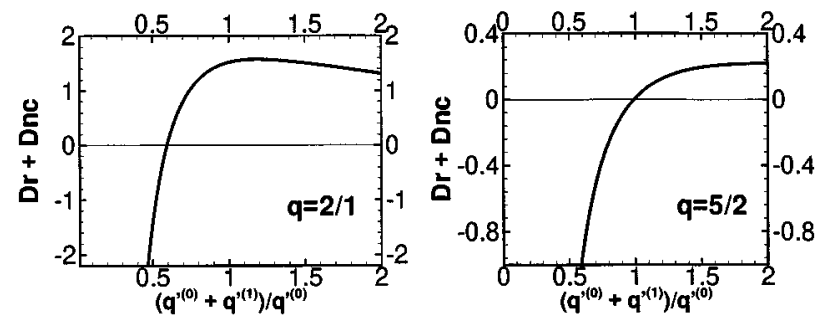

FIG. 10. Pegasus stability curves for two inner rational surfaces for the equilibrium shown in Fig. 1. For the 2/1 surface, when the local shear is decreased by $50 \%$, the pressure drives may be eliminated. The $5 / 2$ rational surface is near marginal stability. 


\section{SUMMARY}

The geometrical dependence of the pressure drive mechanisms for the nonideal MHD tearing mode has been investigated. For large aspect ratio, circular cross-section tokamaks, the resistive interchange term, which is typically a stabilizing term, is generally negligible. For a large aspect ratio, shaped tokamak, the resistive interchange term is more significant. However, in this regime of moderate shear and relatively low beta the resistive interchange and bootstrap terms scale have opposite signs but scale similarly with shaping, so that shaping has very little influence on the drive for these modes. At low aspect ratio where the shear is low and the beta is high, the resistive interchange term is comparable to the bootstrap term and the effect of shaping on the pressure drives is due primarily to its effect on magnetic shear. In this regime, the stability of the modes is very sensitive to the equilibrium profiles used.

To further the understanding of the profile sensitivity, a Greene-and-Chance-like analysis was performed. The analysis reveals that it may be possible to stabilize the modes in LART's by shaping and locally flattening the $q$ profile at low-order rational surfaces via localized current drive. In large aspect ratio cases it would be more difficult to stabilize the modes via locally flattening the $q$ profile. To more fully understand the physics of shaping and aspect ratio on magnetic island formation, a number of other effects need to be addressed. In particular, geometrical effects should also influence $\Delta^{\prime}$. Additionally, it has been suggested that the source of the required seed island perturbation to initiate the neoclassical island growth comes from the geometric coupling from some other MHD event. We leave the examination of these effects to future work.

\section{ACKNOWLEDGMENTS}

The authors wish to thank Bob Miller for generously supplying and providing assistance with the TOQ equilibria code used in this study. We also wish to thank Zuoyang Chang for the TFTR equilibria data and Rob LaHaye for the DIII-D data.

This research is supported by the United States Department of Energy (DOE) under Grants No. DE-FG0286ER53218 and No. DE-FG02-92ER54139.

${ }^{1}$ A. D. Turnbull, T. S. Taylor, E. J. Strait, S. J. Thompson, M. S. Chu, J. R. Ferron, R. J. La Haye, L. L. Lao, R. T. Snider, B. W. Rice, D. Wroblewski, O. Sauter, M. E. Mauel, A. Popov, N. Popova, D. J. Lightly, and J. D. Williams, in Proceedings of the 15th International Conference on Plasma Physics and Controlled Nuclear Fusion Research, Seville (International Atomic Energy Agency, Vienna, 1995), Vol. 1, p. 705.

${ }^{2}$ E. Fredrickson, S. Batha, M. G. Bell, R. V. Budny, C. E. Bush, Z. Chang, C. Z. Cheung, D. S. Darrow, J. Dunlap, G. Y. Fu, H. W. Herrmann, H. Hsuan, R. Majeski, D. K. Mansfield, E. Mazzucato, K. M. McGuire, D. R. Mikkelsen, M. Murakimi, R. Nazikan, A. C. Janos, C. K. Philips, S. A. Sabbagh, G. L. Schmidt, S. D. Scott, J. D. Strachan, E. J. Synakowski, H. Takahashi, G. Taylor, J. R. Wilson, K. L. Wong, M. C. Zarnstorff, S. J. Zweben, and the TFTR team, "TAE modes and MHD activity in TFTR DT Plasmas," in Ref. 1, p. 275.
${ }^{3}$ Z. Chang, E. D. Fredrickson, J. D. Callen, K. M. McGuire, M. G. Bell, R. V. Budny, C. E. Bush, D. S. Darrow, A. C. Janos, L. C. Johnson, H. K. Park, S. D. Scott, J. D. Strachan, E. J. Synakowski, G. Taylor, R. M. Wieland, M. C. Zarnstorff, S. J. Zweben, and the TFTR team, Nucl. Fusion 34, 1309 (1994).

${ }^{4}$ H. P. Furth, J. Killeen, and M. N. Rosenbluth, Phys. Fluids 6, 459 (1963).

${ }^{5}$ See National Technical Information Service Document No. DE6008946 (W. X. Qu and J. D. Callen, "Nonlinear growth of a single neoclassical MHD tearing mode in a tokamak," U. W. Plasma Report 85-5, 1985.) Copies may be ordered from the National Technical Information Service, Springfield, VA 22161

${ }^{6}$ R. Carrera, R. D. Hazeltine, and M. Kotschenreuther, Phys. Fluids 29, 899 (1986).

${ }^{7}$ C. C. Hegna and J. D. Callen, Phys. Fluids 4, 4072 (1992).

${ }^{8}$ Z. Chang, J. D. Callen, E. D. Fredrickson, R. V. Budny, C. C. Hegna, K. M. McGuire, M. C. Zarnstorff, and the TFTR team, Phys. Rev. Lett. 74, 4663 (1995).

${ }^{9}$ R. J. LaHaye, J. D. Callen, M. S. Chu, S. Deshpande, T. A. Gianakon, C. C. Hegna, S. Jardin, L. L. Lao, J. Manikam, D. A. Monticello, A. Pletzer, A. H. Reiman, O. Sauter, E. J. Strait, T. S. Taylor, A. D. Turnbull, and H. R. Wilson, "Practical beta limit in ITER-shaped discharges in DIII-D and its increase by higher collisionality," to be published in Plasma Physics and Controlled Nuclear Fusion Research 1996, Montreal (International Atomic Energy Agency, Vienna, 1997), Paper AP1-21.

${ }^{10}$ H. Zohm, M. Alexander, R. Buttery, S. dePena Hempel, O. Gruber, T. C. Hender, T. Kass, M. Maraschek, W. Sandmann, S. Sesnic, M. Sokoll, A. Stabler, H. R. Wilson, H. P. Zehrfeld, the ASDEX Upgrade-Team, and the NBI-Team, " $\beta$-limiting phenomena in ASDEX Upgrade," to appear in Proceedings of the 23rd EPS Conference on Controlled Fusion and Plasma Physics, Kiev, Ukraine, 1996 (European Physical Society, PetitLancy, in press).

${ }^{11}$ D. A. Gates, B. Lloyd, A. W. Morris, G. McArdle, M. O'Brien, M. Valovic, C. D. Warrick, H. R. Wilson, and the COMPASS-D and the ECRH Teams, " $\beta$-limit studies and the effect of error fields at low collisionality on Compass-D," to appear in Ref. 9, Paper AP1-17.

${ }^{12}$ O. Sauter, R. J. La Haye, Z. Chang, D. A. Gates, Y. Kamada, H. Zohm, A. Bondeson, D. Boucher, J. D. Callen, M. S. Chu, T. A. Gianakon, O. Gruber, R. W. Harvey, C. C. Hegna, L. L. Lao, D. A. Monticello, F. Perkins, A. Pletzer, A. H. Reiman, M. Rosenbluth, E. J. Strait, T. S. Taylor, A. D. Turnbull, F. Waelbroeck, J. C. Wesley, H. R. Wilson, and R. Yoshino, Phys. Plasmas 4, 1654 (1997).

${ }^{13}$ M. Kotschenreuther, R. D. Hazeltine, and P. J. Morrison, Phys. Fluids 28, 294 (1985).

${ }^{14}$ A. H. Glasser, J. M. Greene, and J. L. Johnson, Phys. Fluids 18, 875 (1975)

${ }^{15}$ P. H. Rutherford, Phys. Fluids 16, 1903 (1973).

${ }^{16}$ R. Fitzpatrick, Phys. Plasmas 2, 825 (1995); and L. E. Zakharov (private communication).

${ }^{17}$ T. A. Gianakon, C. C. Hegna, and J. D. Callen, Phys. Plasmas 5, 4637 (1996).

${ }^{18}$ N. N. Gorelenkov, R. V. Budny, Z. Chang, M. V. Gorelenkova, and L. E. Zakharov, Phys. Plasmas 3, 3379 (1996).

${ }^{19}$ H. R. Wilson, J. W. Connor, R. J. Hastie, and C. C. Hegna, Phys. Plasmas 3, 248 (1996).

${ }^{20}$ J. D. Callen, W. X. Qu, K. D. Siebert, B. A. Carreras, K. C. Shaing, and D. A. Spong, in Plasma Physics and Controlled Nuclear Fusion Research 1986, Kyoto (International Atomic Energy Agency, Vienna, 1987), Vol. 2, p. 157.

${ }^{21}$ S. P. Hirshman, Phys. Fluids 31, 3150 (1988).

${ }^{22}$ F. L. Hinton and R. D. Hazeltine, Rev. Mod. Phys. 15, 116 (1976).

${ }^{23}$ Y. R. Lin-Liu and R. L. Miller, Phys. Plasmas 2, 1666 (1995).

${ }^{24}$ J. E. Menard, S. C. Jardin, C. E. Kessel, and J. Manickam, Nucl. Fusion 37, 595 (1997)

${ }^{25}$ R. L. Miller, Y. R. Lin-Liu, A. D. Turnbull, V. S. Chan, L. D. Pearlstein, O. Sauter, and L. Villard, Phys. Plasmas 4, 1062 (1997).

${ }^{26}$ J. M. Greene and M. Chance, Nucl. Fusion 21, 453 (1981). 